\title{
Higiene bucal en la población de San Francisco, Colombia, y sus factores relacionados
}

Dorian Ospina, Est. ${ }_{1}$, Yessid Herrera, Est. ${ }_{1}$, Juan Betancur, Est. ${ }_{1}$, Héctor Bayron Agudelo, MSc. ${ }_{2}$, Adriana Posada López ${ }^{\star}, \mathrm{MSc}_{1}$

Universidad de Antioquia, Facultad Nacional de Salud Pública, Medellín, Colombia

${ }_{2}$ Fundación Universitaria Autónoma de las Américas, Facultad de Odontología, Medellín, Colombia

Recibido: 8 de octubre del 2015 Aprobado: 14 de noviembre del 2015

*Autor de correspondencia: Adriana Posada López. Universidad de Antioquia, Facultad de Odontología, Medellín, Colombia. Calle 64 n. ${ }^{\circ}$ 52-59 BI. 31-108, 2 piso. Teléfono: 2196772. Correo electrónico: adriposalo@gmail.com

Cómo citar este artículo: Ospina D, Herrera Y, Betancur J, Agudelo HB, Posada A. Higiene bucal en la población de San Francisco, Colombia, y sus factores relacionados. Rev Nac Odontol. 2016;12(22):23-30. doi: http://dx.doi.org/10.16925/ od.v12i22.1203

Resumen. Introducción: este artículo describe la higiene bucal de la población de la cabecera municipal de San Francisco, Antioquia (Colombia), según sus condiciones sociodemográficas. Métodos: se realizó un estudio transversal de una muestra no probabilística de la zona urbana del municipio, en personas que se encontraban en las edades trazadoras recomendadas por la Organización Mundial de la Salud $(5,12,15,25,35,45,55$ y 65 años). Se diseñó un instrumento de recolección para indagar por las condiciones sociodemográficas, los hábitos saludables y las variables que permiten estimar el Índice de Higiene Oral Simplificado (IHOS). Se realizó un análisis observacional analítico. Resultados y discusión: la mayor proporción de los encuestados eran mujeres (65\%), con mayor presencia de población en la edad de la infancia. La mayoría pertenecen al régimen subsidiado y residen en estrato bajo. Solo una quinta parte de los encuestados tenían nivel de estudios secundario o superior. El uso del cepillo y la crema dental fue generalizado, en contraste con el uso poco frecuente de la seda dental y el enjuague bucal. En la mayoría de las edades, se evidenció alta prevalencia de higiene oral inadecuada y asociación estadísticamente significativa con el grado de escolaridad. Conclusiones: la higiene oral en el área urbana estuvo relacionada principalmente con el nivel escolar, por lo tanto, los esfuerzos por implementar estrategias educativas que apunten a la promoción de la salud bucal en el municipio deberían estar dirigidos de manera prioritaria para aquellos que están en situación alta de vulnerabilidad.

Palabras clave: factores sociodemográficos, hábitos de salud bucal, odontología comunitaria, población vulnerable. 


\title{
Oral Hygiene in San Francisco's Population (Colombia) and Related Factors
}

\begin{abstract}
Introduction: this article describes oral hygiene of the population in the municipal capital of San Francisco, Antioquia (Colombia), according to their sociodemographic conditions. Methods: a transversal study was conducted of non-probabilistic samples in the municipality's urban area with patients around the investigative ages recommended by the World Health Organization (5, 12, 15, 25, 35, 45, 55 and 65 years). A collecting tool was designed to inquire the sociodemographic conditions, the health habits and the variables that allow an estimation of the Simplified Oral Hygiene Index (sohi). An analytical and observational study was performed. Results and discussion: the higher proportion of survey respondents were women (65\%), with a higher presence of population at the age of childhood. Most of them are in the subsidized system and live in lower stratums. Only a fifth of the respondents have a secondary or higher level of education. The use of toothbrushes and toothpaste was generalized, as opposed to the rare use of dental floss and mouthwash. At most ages, it became evident a higher incidence of inadequate oral hygiene and a significant statistical association to the level of schooling. Conclusion: oral hygiene in urban areas is mainly related to the level of schooling, so initiatives to implement educational strategies that promote oral healthcare at the municipality should be directed to give priority to people in the most vulnerable situations.
\end{abstract}

Keywords: sociodemographic factors, oral healthcare habits, communitarian odontology, vulnerable population.

\section{Higiene bucal na população de San Francisco, Colômbia, seus fatores relacionados}

Resumo. Introdução: este artigo descreve a higiene bucal da população do município de San Francisco, Antioquia (Colômbia), segundo suas condições sócio-demográficas. Métodos: foi feito um estudo transversal de uma amostra não probabilística da zona urbana do município, em pessoas em idades marcadoras recomendadas pela Organização Mundial da Saúde (5, 12, 15, 25, 35, 45, 55 e 65 anos). Foi projetado um instrumento de coleta para indagar pelas condições sócio-demográficas, os hábitos saudáveis e as variáveis que permitem calcular o Índice de Higiene Oral Simplificado (IHOs). Uma análise observacional analítica foi feita. Resultados e discussão: a maior proporção dos pesquisados eram mulheres (65\%), com maior presença de população na idade da infância. A maioria pertence ao regime subsidiado de saúde e são de baixa renda. Apenas uma quinta parte dos pesquisados tinham nível de ensino secundário ou superior. O uso da escovação e o creme dental foi generalizado, em contraste com o uso pouco frequente da seda dental e o enxague bucal. $\mathrm{Na}$ maioria das idades houve alta prevalência de higiene bucal inadequada e associação estatisticamente significativa com o nível de escolaridade. Conclusões: a higiene bucal na zona urbana esteve relacionada principalmente com o nível escolar, portanto, os esforços por implementar estratégias educativas voltadas à promoção da saúde bucal no município deveriam estar direcionados prioritariamente para aqueles em situação de alta vulnerabilidade.

Palavras-chave: fatores sócio-demográficas, hábitos de saúde bucal, odontologia comunitária, população vulnerável. 


\section{Introducción}

En el mundo la prevalencia de caries dental y de la enfermedad periodontal va en aumento. En el caso de la caries, entre el $80 \%$ y el $90 \%$ de la población de la mayoría de los países la ha padecido; los valores más altos se presentan en los países industrializados y en algunos de América Latina, mientras que los niveles son mucho más bajos en los países desarrollados. En países europeos como Rusia, Italia y España; en América, Argentina, Costa Rica y México; y en África, Marruecos y Argelia poseen un índice CPOD (suma de dientes permanentes cariados, perdidos y obturados) moderado; por otro lado, países como Suecia, Francia, Alemania y Reino Unido, en Europa, y Estados Unidos, Brasil y Colombia, en América, son algunos de los que a nivel mundial tienen los índices más altos de caries [1].

En Colombia, el II Estudio de Morbilidad Oral se realizó entre 1977 y 1980, y se encontró que el $96,7 \%$ de la población tiene historia de caries, con un promedio de 12,7 dientes afectados. Casi 20 años después, en 1999, se llevó a cabo el III Estudio Nacional de Salud Bucal (ENSAB III), el cual buscó identificar necesidades y brindar información en materia de conocimientos y prácticas respecto a la salud bucal y sus determinantes. En dicho estudio se reportó una mejoría en niños y jóvenes, dado que el promedio de dientes afectados disminuyó y el de dientes sanos aumentó, aunque, por el contrario, el estado de salud bucal en los adultos se vio afectado negativamente [2]. El último Estudio Nacional de Salud Bucal (ENSAB IV), realizado en el 2014, muestra que, en los dientes permanentes, la prevalencia de caries ha disminuido, que el número de dientes obturados ha aumentado, al igual que los dientes que se pierden por caries, sobre todo en los adultos, y que la zona central de Colombia es la que muestra los resultados más bajos de los obtenidos en el estudio [3].

En relación con lo anterior, la oms, la Federación Dental Internacional y la Asociación Internacional para la Investigación Dental establecieron en el 2013 las nuevas metas globales en salud bucal para el 2020, en las que se planteó que cada región debe disminuir los indicadores de morbilidad bucal según su situación de salud [4]. En Colombia, el Plan Decenal de Salud Pública contempla dentro de sus dimensiones los planes de beneficios, que cuentan con acciones para la educación y atención de eventos que afectan la salud bucal. Dichas acciones son los procedimientos de protección específica, las actividades de educación individual y colectiva y un amplio número de procedimientos para la atención de las condiciones que se presentan en las diferentes edades y grupos poblacionales [5].

Para poder mejorar los indicadores de salud, es necesario intervenir los servicios de salud y otros determinantes, como los factores biológicos, ambientales y de estilos de vida, lo que sugiere un proceso de promoción de la salud y prevención de la enfermedad en las comunidades, y esto implicaría desarrollar actividades de educación para la salud, para lo cual es necesario conocer las características propias de la población a tratar [5].

En el caso particular de este estudio, la población estudiada es la del municipio de San Francisco, ubicado en el oriente de Antioquia. Según un informe del gobernador de turno, durante los años noventa, este municipio fue uno de los más golpeados por la violencia, lo cual ocasionó un fenómeno de desplazamiento de 11500 personas (aprox.) [6]. Según el Censo Nacional del 2005, esta población exhibió una situación de vulnerabilidad dado que el $25 \%$ de la población no sabía leer ni escribir, cerca del 50\% solo había alcanzado el nivel de básica primaria y el $25 \%$ restante no tenía nivel educativo alguno [7].

Teniendo en cuenta lo anterior, el objetivo de este estudio fue describir la higiene bucal de la población de la cabecera municipal de San Francisco, Antioquia, en edades trazadoras, de acuerdo con las condiciones sociodemográficas.

\section{Métodos}

Se realizó un estudio transversal en el que se incluyeron los residentes de la zona urbana del municipio de San Francisco, Antioquia. La unidad de análisis se enfocó en las personas con las edades trazadoras recomendadas por la oms $(5,12,15,25$, 35, 45, 55 y 65 años). En total, se encuestaron 145 individuos que se encontraban en sus hogares en el momento de la visita. Se diseñó un instrumento de recolección que indagó por las variables sociodemográficas, los hábitos de higiene y el IHos.

En cuanto a las variables sociodemográficas, se tuvo en cuenta: edad, sexo, régimen de afiliación, nivel de alfabetismo, estrato socioeconómico, nivel 
académico, nivel de ingresos. En relación con los hábitos saludables, se tuvo en cuenta: número de veces que se cepilla al día y a la semana, última vez que compró cepillo de dientes, número de veces que usa la seda dental, uso del enjuague bucal, uso de la crema dental, cepillado de la lengua, última vez que recibió atención odontológica, razón de la última atención odontológica y percepción al ir al dentista.

Para efecto del análisis, se calculó el iHos [8], el cual mide la presencia de placa blanda y dura en dientes deciduos y permanentes. Dicho indicador se categorizó en nivel adecuado de higiene (IHOS $\leq$ 0,6), y nivel inadecuado de higiene ( HOS $>0,6)$.

La información se recolectó el 19 y el 20 de noviembre del 2014, y fue precedida por una estandarización, calibración y la prueba piloto para verificar el instrumento y asegurar la comprensión de las preguntas y la consistencia general. El examen bucal fue realizado por odontólogos que se desplazaron al municipio, según las directrices de la oMs, para la realización de índices bucodentales.

El análisis descriptivo se realizó mediante el cálculo de medidas de dispersión y posición para las variables cuantitativas. Para el caso de las variables cualitativas, se obtuvieron frecuencias y porcentajes, y para establecer las variables que se asociaban estadísticamente con el IHOS, se aplicó la prueba de Ji-cuadrado. Se asumió como asociación significativa aquella cuyo valor " $p$ " fuera menor a 0,05. Para el procesamiento y análisis de los datos, se utilizaron los software Microsoft Office Excel y Access $^{\oplus}$ 2013, y el software estadístico IBM SPSs v20.

Este estudio fue avalado por la administración local del momento; además, se contemplaron los protocolos de examen odontológico, asentimiento y consentimiento informado, protección de la identidad y la veracidad de la información. Esta investigación cumple con la normativa internacional (Declaración de Helsinki) [9] y nacional (Resolución 8430 del Ministerio de Salud y Protección Social) [10] para investigación en salud, considerándose como un estudio de riesgo mínimo.

\section{Resultados}

Del total de encuestados, el $65 \%$ era mujeres. La mayor proporción de participantes tenía entre $5 \mathrm{y}$ 12 años (36,9\%). Cerca del 95\% de los individuos residía en viviendas en estrato socioeconómico bajo. En cuanto al aseguramiento, alrededor del $71 \%$ pertenecía al régimen subsidiado. Respecto al nivel educativo, el $81 \%$ no superaba la primaria (tabla 1).

Tabla 1. Distribución de población según características demográficas. San Francisco, Antioquia, 2014

\begin{tabular}{|c|c|c|c|}
\hline Variable & Categoría & $\mathbf{n}$ & $\%$ \\
\hline \multirow[t]{8}{*}{ Edad en años cumplidos } & 5 & 26 & 18,4 \\
\hline & 12 & 26 & 18,4 \\
\hline & 15 & 18 & 12,8 \\
\hline & 25 & 16 & 11,3 \\
\hline & 35 & 15 & 10,6 \\
\hline & 45 & 15 & 10,6 \\
\hline & 55 & 12 & 8,5 \\
\hline & 65 & 13 & 9,2 \\
\hline \multirow[t]{2}{*}{ Sexo } & Hombre & 50 & 35,5 \\
\hline & Mujer & 91 & 64,5 \\
\hline \multirow[t]{3}{*}{ Régimen de afiliación } & Contributivo & 34 & 25,4 \\
\hline & Subsidiado & 95 & 70,9 \\
\hline & Otro & 5 & 3,7 \\
\hline \multirow[t]{4}{*}{ Alfabetismo } & Sabe leer y escribir & 100 & 70,9 \\
\hline & Solo leer & 3 & 2,1 \\
\hline & Solo escribir & 2 & 1,4 \\
\hline & Ninguna & 36 & 25,5 \\
\hline \multirow[t]{2}{*}{ Estrato recodificado } & Bajo & 128 & 94,8 \\
\hline & Medio & 7 & 5,2 \\
\hline \multirow[t]{4}{*}{ Nivel académico } & Ninguno & 55 & 41,0 \\
\hline & Primaria & 53 & 39,6 \\
\hline & Secundaria & 18 & 13,4 \\
\hline & Técnico-profesional & 8 & 6,0 \\
\hline \multirow[t]{2}{*}{ Nivel de ingresos } & Menos de $\$ 616.600$ & 60 & 81,1 \\
\hline & $\$ 616.600$ o más & 14 & 18,9 \\
\hline
\end{tabular}

Fuente: elaboración propia a partir de la base de datos construida en el marco del proyecto

Cuando se indagó por los hábitos relacionados con la salud oral, todos los encuestados afirmaron practicar el cepillado de los dientes, pero de estos, el $6 \%$ indicó no cepillarse diariamente. El $67 \%$ expresó cepillarse tres veces o más al día y el $48 \%$ no ha cambiado de cepillo hace más de dos meses. El uso de la seda dental es poco frecuente: el $43 \%$ no la usa; en el caso del enjuague bucal, solo lo utiliza el $30 \%$. Por otro lado, el $92 \%$ de las personas expresó cepillarse la lengua.

En cuanto al uso de los servicios de salud, el $61 \%$ respondió no haber ido al odontólogo en los últimos seis meses, y siete de los encuestados 
reportaron no haber asistido ni sola una vez a consulta odontológica. La razón más frecuente de la última atención odontológica fue por control, prevención y revisión rutinaria, mientras un $26 \%$ del total asistieron para tratar caries, dolor o infección. Al 64\% de la población le resulta agradable asistir al odontólogo, y el 14,2\% afirmó que el hecho de asistir al odontólogo le resultaba desagradable (tabla 2).

Tabla 2. Distribución de hábitos de higiene oral en población evaluada. San Francisco, Antioquia, 2014

\begin{tabular}{|c|c|c|c|}
\hline Variable & Categorías & $\mathbf{n}$ & $\%$ \\
\hline \multirow{3}{*}{$\begin{array}{l}\text { Número de veces que } \\
\text { se cepilla a la semana }\end{array}$} & Diariamente & 135 & 94,4 \\
\hline & Entre 4 y 6 veces & 5 & 3,5 \\
\hline & Entre 1 y 3 veces & 3 & 2,1 \\
\hline \multirow{3}{*}{$\begin{array}{l}\text { Número de veces que } \\
\text { se cepilla al día }\end{array}$} & Una vez & 8 & 5,7 \\
\hline & Dos veces & 38 & 27,0 \\
\hline & Tres veces o más & 95 & 67,4 \\
\hline \multirow{4}{*}{$\begin{array}{l}\text { Última vez que com- } \\
\text { pró cepillo de dientes }\end{array}$} & Este mes & 75 & 52,4 \\
\hline & El mes anterior & 27 & 18,9 \\
\hline & Hace dos meses o más & 27 & 18,9 \\
\hline & No lo recuerdo & 14 & 9,8 \\
\hline \multirow{3}{*}{$\begin{array}{l}\text { Estado actual del } \\
\text { cepillo de dientes }\end{array}$} & Si, está en buen estado & 111 & 77,6 \\
\hline & No, está en mal estado & 25 & 17,5 \\
\hline & No sé & 7 & 4,9 \\
\hline \multirow{5}{*}{$\begin{array}{l}\text { Número de veces } \\
\text { al día que usa seda } \\
\text { dental }\end{array}$} & Una sola vez & 31 & 21,7 \\
\hline & Dos veces & 19 & 13,3 \\
\hline & Tres veces o más & 22 & 15,4 \\
\hline & Ninguna vez & 62 & 43,4 \\
\hline & Sin información & 9 & 6,3 \\
\hline \multirow{2}{*}{ Usa enjuague bucal } & $\mathrm{Si}$ & 43 & 30,1 \\
\hline & No & 100 & 69,9 \\
\hline \multirow{2}{*}{ Usa crema dental } & $\mathrm{Si}$ & 141 & 98,6 \\
\hline & No & 2 & 1,4 \\
\hline \multirow{2}{*}{ Se cepilla la lengua } & $\mathrm{Si}$ & 131 & 91,6 \\
\hline & No & 12 & 8,4 \\
\hline \multirow{4}{*}{$\begin{array}{l}\text { Última vez que recibió } \\
\text { atención odontológica }\end{array}$} & Menos de 6 meses & 49 & 34,5 \\
\hline & Entre 6 meses y un año & 38 & 26,8 \\
\hline & Más de un año & 48 & 33,8 \\
\hline & Nunca & 7 & 4,9 \\
\hline \multirow{6}{*}{$\begin{array}{l}\text { Razón de la última } \\
\text { atención odontológica }\end{array}$} & Control y prevención & 28 & 20,3 \\
\hline & Revisión rutinaria & 37 & 26,8 \\
\hline & Tratamiento de ortodoncia & 10 & 7,2 \\
\hline & Caries, dolor o infección & 36 & 26,1 \\
\hline & Endodoncia o exodoncia & 6 & 4,3 \\
\hline & No recuerda & 21 & 15,2 \\
\hline \multirow{4}{*}{$\begin{array}{l}\text { El hecho de ir al odon- } \\
\text { tólogo le resulta }\end{array}$} & Agradable & 90 & 63,8 \\
\hline & Desagradable & 20 & 14,2 \\
\hline & Indiferente & 24 & 17,0 \\
\hline & No sé & 7 & 5,0 \\
\hline
\end{tabular}

Fuente: elaboración propia a partir de la base de datos construida en el marco del proyecto
En cuanto a la distribución del IHos, los participantes en edad de 12 y 55 años tienen las mayores proporciones de nivel inadecuado de higiene (73\% y $75 \%$, respectivamente); en términos generales, los hombres poseen un menor grado de higiene en comparación con las mujeres. Por otro lado, aquellos que pertenece al régimen contributivo y tienen ingresos mensuales superiores al salario mínimo presentan mayor probabilidad de tener una higiene adecuada. Respecto al nivel académico, aquellos que no lograron algún grado de escolaridad presentaron mayor proporción de niveles inadecuados de IHOS; estas diferencias fueron estadísticamente significativas (valor $\mathrm{p}<0,05$; tabla 3 ).

\section{Discusión}

Los resultados de este estudio dan cuenta de que la mayor proporción eran mujeres, lo cual indica que ellas son las que con mayor frecuencia se encuentran en la cabecera de San Francisco y son las directamente encargadas del cuidado del hogar y del trabajo doméstico, característica común de la división del trabajo en países latinoamericanos como Colombia [11]. La mayor parte de la población evaluada estaba en la edad de la infancia, grupo etario considerado como muy importante dentro del conjunto de políticas sociales y en salud, y es por eso que los esfuerzos de la oms y la Unicef se han dirigido a crear diferentes estrategias educativas y de protección que pretenden mejorar la salud de los niños [12].

La mayoría de las personas encuestadas pertenece al régimen subsidiado; el $25 \%$ de la población es analfabeta, lo cual es coherente con que casi el 50\% no tenía ningún nivel de estudios y casi el $100 \%$ pertenecía al estrato bajo. Las disparidades mencionadas son factores que favorecen las inequidades en salud, puesto que son las personas de menos recursos las que tienen más posibilidades de sufrir enfermedades, que no solo son el resultado del proceso morboso como tal, sino también de la interacción del individuo con su entorno económico, político y social. Los resultados mencionados muestran la presencia del gradiente social en salud, según el cual, a medida que se baja un peldaño en la escalera social, aumenta la prevalencia de todo tipo de enfermedades y problemas de salud en una comunidad [13]. 
Tabla 3. Nivel de higiene variables sociodemográficas de la población encuestada. San Francisco, Antioquia, 2014

\begin{tabular}{|c|c|c|c|c|c|c|}
\hline \multirow{3}{*}{ Variable } & \multirow{3}{*}{ Categoría } & \multicolumn{4}{|c|}{ IHOS } & \multirow[b]{3}{*}{ valor $\mathrm{p}^{*}$} \\
\hline & & \multicolumn{2}{|c|}{ Adecuada } & \multicolumn{2}{|c|}{ Inadecuado } & \\
\hline & & $\mathbf{n}$ & $\%$ & $\mathbf{n}$ & $\%$ & \\
\hline \multirow{8}{*}{$\begin{array}{l}\text { Edad en } \\
\text { años } \\
\text { cumpli- } \\
\text { dos }\end{array}$} & 5 & 8 & 33,3 & 16 & 66,7 & \\
\hline & 12 & 7 & 26,9 & 19 & 73,1 & \\
\hline & 15 & 5 & 29,4 & 12 & 70,6 & \\
\hline & 25 & 4 & 28,6 & 10 & 71,4 & \\
\hline & 35 & 7 & 50,0 & 7 & 50,0 & \\
\hline & 45 & 7 & 50,0 & 7 & 50,0 & \\
\hline & 55 & 2 & 25,0 & 6 & 75,0 & \\
\hline & 65 & 0 & 0,0 & 4 & 100,0 & \\
\hline \multirow{2}{*}{ Sexo } & Hombre & 13 & 28,9 & 32 & 71,1 & 0,54 \\
\hline & Mujer & 27 & 34,2 & 52 & 65,8 & \\
\hline \multirow{3}{*}{$\begin{array}{l}\text { Régimen } \\
\text { de afilia- } \\
\text { ción }\end{array}$} & $\begin{array}{l}\text { Contribu- } \\
\text { tivo }\end{array}$ & 10 & 35,7 & 18 & 64,3 & 0,77 \\
\hline & Subsidiado & 25 & 39,8 & 59 & 70,2 & \\
\hline & Otro & 2 & 40,0 & 3 & 60,0 & \\
\hline \multirow{2}{*}{$\begin{array}{l}\text { Estrato } \\
\text { socioeco- } \\
\text { nómico }\end{array}$} & Bajo & 38 & 33,9 & 74 & 66,1 & 0,38 \\
\hline & Medio & 1 & 16,7 & 5 & 83,3 & \\
\hline \multirow{4}{*}{$\begin{array}{l}\text { Nivel de } \\
\text { estudios }\end{array}$} & Ninguno & 8 & 17,8 & 37 & 82,2 & 0,03 \\
\hline & Primaria & 18 & 34,6 & 34 & 65,4 & \\
\hline & Secundaria & 8 & 57,1 & 6 & 42,9 & \\
\hline & $\begin{array}{l}\text { Técni- } \\
\text { co-profe- } \\
\text { sional }\end{array}$ & 3 & 50,0 & 3 & 50,0 & \\
\hline \multirow{2}{*}{$\begin{array}{l}\text { Nivel de } \\
\text { ingresos }\end{array}$} & $\begin{array}{l}\text { Menos de } \\
\$ 616.600\end{array}$ & 14 & 26,4 & 39 & 73,6 & 0,62 \\
\hline & $\begin{array}{l}\$ 616.600 \text { o } \\
\text { más }\end{array}$ & 4 & 33,3 & 8 & 66,7 & \\
\hline
\end{tabular}

Fuente: elaboración propia a partir de la base de datos construida en el marco del proyecto

Al comparar los resultados obtenidos con los que se presentan en el ENSAB III y el ENSAB IV, y con un estudio realizado en el Amazonas en 80 personas, se encuentran similitudes en cuanto a los hábitos de higiene que tienen los habitantes de San Francisco, en los que el uso del cepillo y la crema dental es generalizado y con la frecuencia recomendada por los odontólogos e higienistas [3, 14], lo que refleja los posibles procesos educativos que se brindan en la comunidad a través de legados culturales útiles para la apropiación del autocuidado y el cambio de comportamiento hacia estilos de vida saludable, lo que Antonovsky llama salutogénesis, según la cual los individuos se convierten en sujetos responsables, activos y participantes de su cuidado $[15,16]$. Lo anterior contrasta con el uso poco frecuente de la seda dental y del enjuague bucal $[3,14]$, implementos que, según la literatura, ayudarían a desorganizar la placa bacteriana en cavidad bucal y evitar la incidencia de enfermedades orales [17].

En cuanto al acceso a los servicios de salud, se observó que la mitad de la población ha recibido atención en el servicio odontológico hace menos de un año y lo hacen en especial por control y revisión, o porque sienten dolor, y la mayoría percibe el hecho de ir al odontólogo como agradable, lo quetienequeverconlosdiferentesdeterminantesque influyen en el acceso y la utilización de los servicios de salud, en este caso, la odontología. Dichos determinantes son conceptualizados como factores individuales, sociales y estructurales o propios del sistema de servicios de salud $[18,19]$.

Al medir el IHos, es relevante considerar que en la mayoría de las edades predominó la higiene oral inadecuada, lo cual indica que los esfuerzos en la prevención se deben enfocar por igual hacia los diferentes grupos poblacionales. Lo anterior tiene diferencias con un estudio realizado en la población universitaria de México, en el que se encontró que el $81,5 \%$ de los encuestados tenían un índice de higiene oral adecuada, mientras que en nuestro estudio solo el $27,6 \%$ de los encuestados registró una higiene oral adecuada [20], posiblemente por el nivel de estudios que tenían los participantes; nuestros resultados muestran diferencias estadísticamente significativas en la higiene oral cuando se relaciona con el nivel de estudios. Respecto al sexo, en un estudio realizado en población escolar en el municipio de Jericó, Antioquia, se encontró que el promedio del inos es mucho menor en mujeres $(1,25)$ respecto a los hombres $(1,50)$ y concuerda con nuestros resultados ya que estos nos permiten aseverar que los hombres poseen un menor grado de higiene en comparación con las mujeres, lo cual se explica por el interés que tienen las mujeres por el cuidado de su salud [21,22].

La literatura sugiere que las condiciones de vida de los habitantes - como vivir en un estrato socioeconómico bajo y no tener estudios- demuestran situaciones de desigualdad social, sobre todo en aquellos que han sido víctimas de la violencia, lo que redunda en una afección en la salud de aquellos que la han padecido, ocasionando una atención especial por parte del Estado [23, 24, 25].

Por último, una de las fortalezas de este estudio consiste en que es la primera aproximación poblacional para evaluar la presencia de la placa bacteriana en el municipio de San Francisco, como 
principal factor de riesgo para enfermedades como la caries y la enfermedad periodontal. Otra fortaleza es que el estudio surge desde la inquietud investigativa que se promueve en los cursos de investigación formativa del programa Gerencia en Sistemas de Información, de la Facultad Nacional de Salud Pública de la Universidad de Antioquia, lo que permitió el contacto con el sector político y con personal del área de la salud como los odontólogos, situación que suscita un aprendizaje significativo a partir de una realidad. Como limitación, se hace necesario mencionar que los resultados de este estudio se deben analizar con mesura, teniendo en cuenta que los datos fueron recogidos en la cabecera municipal, y no en la zona rural, por lo que la evaluación de la higiene oral como principal factor de riesgo puede estar por encima o por debajo de lo realmente estimado. Además, no es recomendable realizar extrapolaciones o generalizaciones al total del territorio, dado que las personas fueron elegidas de forma no probabilística. Sin embargo, la información encontrada da visos del estado de salud bucal de la población y sirve como base para posteriores estudios de mayor alcance.

\section{Conclusiones}

La higiene oral de los habitantes de San Francisco está relacionada principalmente con el nivel de estudios de las personas, lo que indica que se deben implementar estrategias educativas que apunten a la promoción de la salud y a la prevención de la enfermedad para aquellos que están en situación alta de vulnerabilidad, en este caso por sus antecedentes de violencia, lo que puede redundar en bajos niveles de escolaridad. Es necesario realizar una aproximación más detallada de los elementos que inciden en la falta de acceso a los servicios de salud, tanto de la población urbana como de la rural, con el fin de mostrar un panorama amplio de la situación del municipio, máxime cuando ha sido permeado por la violencia.

Es necesario que los municipios conozcan su realidad particular, para lo cual se debe tener la voluntad política para realizar diagnósticos en salud que orienten planes de intervención eficaces, pertinentes y oportunos, orientados por los estudios poblacionales como el Iv Estudio Nacional de Salud bucal, con el fin de contribuir al mejoramiento de la salud bucal de las personas, de su salud general y de su calidad de vida.

\section{Agradecimientos}

Los autores agrademos a los habitantes de la cabecera del municipio de San Francisco, Antioquia, por permitir el acercamiento de los examinadores; al personal administrativo de dicho municipio por suministrar la información requerida y por la disposición para realizar el trabajo de campo; a los profesores Jesús Ernesto Ochoa y Tomás Bernal por el acompañamiento durante la ejecución de proyecto. Además, queremos reconocer el trabajo de los estudiantes de Gerencia en Sistemas de Información y de Odontología de la Universidad de Antioquia.

\section{Referencias}

[1] Petersen E, Bourgeois D, Ogawa H, Estupinan-Day $S$, Ndiaye C. The global burden of oral diseases and risks to oral health. Bulletin of the World Health Organization. 83. 661-9. [Internet]. Disponible en: http://www.scielosp.org/scielo.php?script=sci_arttext\&pid=S0042-96862005000900011\&lng=en\&n$\mathrm{rm}=\mathrm{iso} \& \operatorname{tng}=\mathrm{en}$

[2] Colombia. Ministerio de Salud. III Estudio Nacional de Salud Bucal (eNSAB III). Colombia. [Internet]. 1999. Disponible en: http://visitaodontologica. CO/ARCHIVOS/ARCHIVOS-NORMAS/Salud\%20PubliCa_P_Y_P/II_ESTUDIO_NACIONAL_SALUD_BUCAL. pdf

[3] República de Colombia. Ministerio de Salud. Iv Estudio Nacional de Salud Bucal (ENSAB IV). [Internet]. 2014. Disponible en: https://www.minsalud. gov.co/sites/rid/Lists/BibliotecaDigital/RIDE/vs/PP/ ENSAB-IV-Situacion-Bucal-Actual.pdf .

[4] Hobdell M, Petersen PE, Clarkson J, Johnson N. Global goals for oral health 2020. Int Dent J. 2003;53(5):285-8. doi: http://dx.doi.org/10.1111/ j.1875-595x.2003.tb00761.x.

[5] Espinoza-Gonzáles L. Cambios del modo y estilo de vida; su influencia en el proceso salud-enfermedad. Rev Cub Estomatol. 2004;41(3).

[6] Gobernación de Antioquia. En San Francisco se puede ver y tocar la esperanza. [Internet]. 2013. Disponible en: http://www.antioquia.gov.co/index.php/prensa/historico/15398-en-san-francisco-se-puede-ver-y-tocar-la-esperanza/ 
[7] Municipio de San Francisco. Plan de Desarrollo Municipio de San Francisco 2012-2015, San Francisco Oportunidades para todos. [Internet]. Disponible en: http://antioquia.gov.co/Plan_de_desarrollo_2012_2015/Anteproye ctos_Municipales_ de_desarrollo/orIENTE/San_Francisco.pdf

[8] Greene JC, Vermillion JR. The simplified oral hygiene index. J Am Dent Assoc. 1964;68:7-13. doi: http://dx.doi.org/10.14219/jada.archive.1964.0034.

[9] World Medical Association General Assembly. World Medical Association Declaration of Helsinki: ethical principles for medical research involving human subjects. J Intern Bioet. 2004;15(1):124-9. doi: http://dx.doi.org/10.3917/jib.151.0124.

[10] República de Colombia. Ministerio de Salud. Resolución nº 008430 de 1993 (4 de octubre de 1993). Por la cual se establecen las normas científicas, técnicas y administrativas para la investigación en salud. Bogotá: El Ministerio; 1993.

[11] Gómez E. Género, equidad y acceso a los servicios de salud: una aproximación empírica. Rev Panam Salud Pub. 2002;11(5-6) [Internet]. Disponible en: http://www.scielosp.org/pdf/rpsp/v11n5-6/10718. pdf. doi: http://dx.doi.org/10.1590/s1020-4989200 2000500008.

[12] Gove S. Integrated Management of Childhood Illness by Outpatient Health Workers: Technical Basis and Overview. The who Working Group on Guide lines for Integrated Management of the Sick Child. Bull World Health Organ. 1997;75(Suppl 1):7-24.

[13] Álvarez L. Los determinantes sociales de la salud: más allá de los factores de riesgo. Rev Gerenc Polit Salud. 2009;8(17):69-79.

[14] Molina YP, Torres MH, Escalante ADP. Caracterización de la condición periodontal, hábitos y costumbres en salud oral en indígenas de comunidades amazónicas de Colombia: Tarapacá, Amazonas. Acta Odontol Col. 2012;2(2);77-91.

[15] Rivera F, Ramos P, Moreno C, García M. Análisis del modelo salutogénico en España: aplicación en salud pública e implicaciones para el modelo de activos en salud. Rev Esp Salud Pública.
2011;85(2):129-39. [Internet]. Disponible en: http:// scielo.isciii.es/scielo.php?script $=$ sci_arttext\&pi$\mathrm{d}=$ S1135-57272011000200002\&lng=es.

[16] Álvarez-Dardet C, Ruiz MT. Patrimonio de salud: ¿son posibles las políticas salutogénicas? Rev Esp Salud Publica. 2011;85(2):123-7. [Internet]. Disponible en: http://scielo.isciii.es/scielo.php? script=sci arttext\&pid=S1135-57272011000200001\&lng=es.

[17] Soria-Hernández MA, Molina N. Rodríguez-P R. Hábitos de higiene bucal y su influencia sobre la frecuencia de caries dental. Rev Acta Pediatr. Mex 2008;29(1):21-4.

[18] Aday LA, Andersen R. A Framework for the Study of Access to Medical Care. Health Serv Res. 1974;9(3):208-20.

[19] Hirmas M, Poffald L, Jasmen AM, Aguilera X, Delgado I, Vega J. Barreras y facilitadores de acceso a la atención de salud: una revisión sistemática cualitativa. Rev Panam Salud Pública. 2013;33(3):223-9.

[20] Ríos NIG, García MHM. Determinación de los índices CPO-D e IHOs en estudiantes de la Universidad Veracruzana, México. Rev Chil Sal Púb. 2012;16(1):26. doi: http://dx.doi.org/10.5354/07173652.2012.18609.

[21] Mazarro G. Análisis de las diferencias de género en el cuidado bucodental. Gac Dent 2012;241:114-25.

[22] Fukai K, Takaesu Y, Maki Y. Gender differences in oral health behavior and general health habits in an adult population. Bull Tokyo Dent Coll. 1999; 40(4):187-93.

[23] Watt RG. From victim blaming to upstream action: tackling the social determinants of oral health inequalities. Comm Dent Oral Epidemiol. 2007;35(1):1-11.

[24] World Health Organization (wHo). A conceptual framework for action on the social determinants of health: Social Determinants of Health Discussion Paper 2. Geneva: wHo; 2010.

[25] Frohlich KL, Potvin L. Transcending the known in public health practice: the inequality paradox: the population approach and vulnerable populations. Am J Public Health. 2008;98(2):216-21. 